ARTICLE

\title{
MiR-125a targets effector programs to stabilize Treg-mediated immune homeostasis
}

\author{
Wen Pan ${ }^{1,2,3, \star}$, Shu Zhu ${ }^{4, \star}$, Dai Dai ${ }^{1,2, \star}$, Zheng $\mathrm{Liu}^{5}$, Dan $\mathrm{Li}^{6}$, Bin $\mathrm{Li}^{6}$, Nicola Gagliani ${ }^{4}$, Yunjiang Zheng ${ }^{4}$, \\ Yuanjia Tang ${ }^{1,2}$, Matthew T. Weirauch ${ }^{7}$, Xiaoting Chen ${ }^{8}$, Wei Zhu ${ }^{5}$, Yue Wang ${ }^{5}$, Bo Chen ${ }^{5}$, Youcun Qian? \\ Yingxuan Chen ${ }^{9}$, Jingyuan Fang ${ }^{9}$, Ronald Herbst ${ }^{5}$, Laura Richman ${ }^{5}$, Bahija Jallal ${ }^{5}$, John B. Harley ${ }^{7,10}$, \\ Richard A. Flavell ${ }^{4}$, Yihong $\mathrm{Yao}^{5}$ \& Nan Shen ${ }^{1,2,7,11}$
}

Although different autoimmune diseases show discrete clinical features, there are common molecular pathways intimately involved. Here we show that miR-125a is downregulated in peripheral $\mathrm{CD}^{+}{ }^{+} \mathrm{T}$ cells of human autoimmune diseases including systemic lupus erythematosus and Crohn's disease, and relevant autoimmune mouse models. miR-125a stabilizes both the commitment and immunoregulatory capacity of Treg cells. In miR-125adeficient mice, the balance appears to shift from immune suppression to inflammation, and results in more severe pathogenesis of colitis and experimental autoimmune encephalomyelitis (EAE). The genome-wide target analysis reveals that miR-125a suppresses several effector T-cell factors including Stat3, Ifng and II13. Using a chemically synthesized miR-125a analogue, we show potential to re-programme the immune homeostasis in EAE models. These findings point to miR-125a as a critical factor that controls autoimmune diseases by stabilizing Treg-mediated immune homeostasis.

\footnotetext{
${ }^{1}$ State Key Laboratory of Oncogenes and Related Genes, Shanghai Cancer Institute, Renji Hospital, Shanghai Jiaotong University School of Medicine, Shanghai 200032, China. ${ }^{2}$ Joint Molecular Rheumatology Laboratory of the Institute of Health Sciences and Shanghai Renji Hospital, Shanghai Institutes for Biological Sciences, Chinese Academy of Sciences, and Shanghai Jiaotong University School of Medicine, Shanghai 200025, China. ${ }^{3}$ Department of Genetics, Yale University School of Medicine, New Haven, Connecticut 06520, USA. ${ }^{4}$ Department of Immunobiology, Yale University School of Medicine, New Haven, Connecticut 06520, USA. ${ }^{5}$ Medlmmune LLC, Gaithersburg, Maryland 20878, USA. ${ }^{6}$ Institute Pasteur of Shanghai, Chinese Academy of Sciences, Shanghai 200025, China. ${ }^{7}$ Center for Autoimmune Genomics and Etiology (CAGE) and Divisions of Rheumatology, Cincinnati Children's Hospital Medical Center, Cincinnati Ohio 45229, USA. ${ }^{8}$ School of Electronic and Computing Systems, University of Cincinnati, Cincinnati, Ohio 45221, USA. ${ }^{9}$ Division of Gastroenterology and Hepathology, Renji Hospital, Shanghai Institute of Digestive Disease, Shanghai Jiaotong University School of Medicine, Shanghai 200001, China. ${ }^{10}$ US Department of Veterans Affairs Medical Center, Cincinnati, Ohio 45220, USA. ${ }^{11}$ Collaborative Innovation Center of Systems Biomedicine, Shanghai Jiaotong University, Shanghai 200240, China. ${ }^{\star}$ These authors contributed equally to this work. Correspondence and requests for materials should be addressed to N.S. (email: nanshensibs@gmail.com) or to Y.Y. (email: YaoY@medimmune.com).
} 
T he clinical presentation of any specific autoimmune disease is the culmination of complex interactions between genetics, primary and secondary immune effector mechanisms, and environmental triggers ${ }^{1,2}$. Although different autoimmune diseases show discrete clinical features, there are common molecular pathways intimately involved, which ultimately define specific diseases ${ }^{3,4}$. Distinct lineages of $\mathrm{CD}^{+}$ $\mathrm{T}$ cells can function to both drive and constrain immunemediated pathology. While T effector cells are often implicated in multiple autoimmune diseases, Treg cells suppress inflammation and control the development of multiple autoimmune diseases, such as type 1 diabetes, multiple sclerosis and inflammatory bowel disease ${ }^{5-7}$. Foxp3 acts as a lineage specification factor of Treg cells. It binds to multiple genes and plays a dual role as both a transcriptional activator and repressor ${ }^{8}$. Sustained expression of Foxp3 amplifies and stabilizes molecular features of Treg precursor cells. Simultaneously, it represses the molecular features deleterious to Treg cell differentiation or function, thereby preventing deviation of Treg cells into effector T-cell lineages ${ }^{9,10}$.

The extensive involvement of microRNAs (miRNAs) across human diseases helps to understand the pathogenesis of various ailments and might lead to eventual development of effective therapeutics against these diseases ${ }^{11}$. A subset of miRNAs have shown therapeutic promise and are currently actively pursued either in preclinical or clinical trials ${ }^{12,13}$. However, most studies and patents in the miRNA field are related to the treatment of cancer, viral infections, ocular disorders, cardiovascular disorders and neurological disorders. Promising miRNA candidates for therapeutic development in autoimmune diseases are still very limited ${ }^{12}$.

In this study, we show that miR-125a is downregulated in peripheral $\mathrm{CD}^{+} \mathrm{T}$ cells of multiple human autoimmune diseases and in relevant mouse models. We further utilize miR-125a-deficient mice and show that miR-125a stabilizes both the commitment and immunosuppressive capacity of Treg cells. Deficiency of miR-125a results in more severe colitis and encephalomyelitis (EAE) pathogenesis. The genome-wide target analysis reveals that miR-125a suppresses several effector T-cell factors such as Stat3, Ifng and Il13. Moreover, a chemically synthesized miR-125a analogue not only contributes to EAE prevention but also alleviates established EAE. Together, our findings demonstrate miR-125a as a critical regulator that stabilizes Treg-mediated immune homeostasis and controls autoimmunity.

\section{Results}

MiR-125a is downregulated in autoimmune $\mathrm{CD4}^{+} \mathrm{T}$ cells. $\mathrm{CD} 4^{+}$T-helper cells play a variety of important roles in the development and maintenance of various autoimmune diseases. Our miRNA microarray data showed that miR-125a-5p (named 'miR-125a' here) was among top 10 downregulated miRNAs in the splenic $\mathrm{CD}^{+}{ }^{+} \mathrm{T}$ cells isolated from MRL/lpr mouse strain, the most commonly studied mouse model for human lupus at 5 and 16 weeks of age (Supplementary Fig. 1a,c), as well as in the splenic $\mathrm{CD} 4^{+} \mathrm{T}$ cells isolated from different progressive stages of experimental autoimmune EAE, which is a mouse model for human multiple sclerosis (Supplementary Fig. 1b,d). MiR-125a was also the most significantly downregulated miRNA among all commonly altered miRNAs in the two sets of microarray data. Moreover, previous studies have shown that downregulation of miR-125a in peripheral $\mathrm{T}$ cells is associated with human multiple sclerosis $^{14}$. Therefore, we hypothesized that miR-125a might be a commonly dysregulated miRNA in some human autoimmune diseases. To test the hypothesis, we collected samples from

another two different human autoimmune diseases and some mouse models, and confirmed that miR-125a was indeed significantly decreased in disease $\mathrm{CD} 4{ }^{+} \mathrm{T}$ cells compared with healthy $\mathrm{CD} 4^{+} \mathrm{T}$ cells. These samples included 22 patients with systemic lupus erythematosus (SLE) versus 20 healthy controls

a
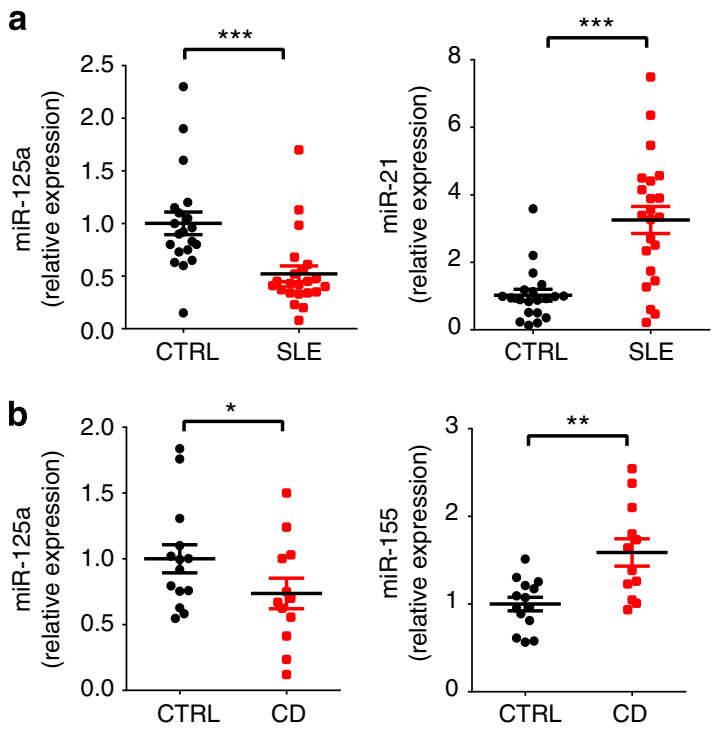

C
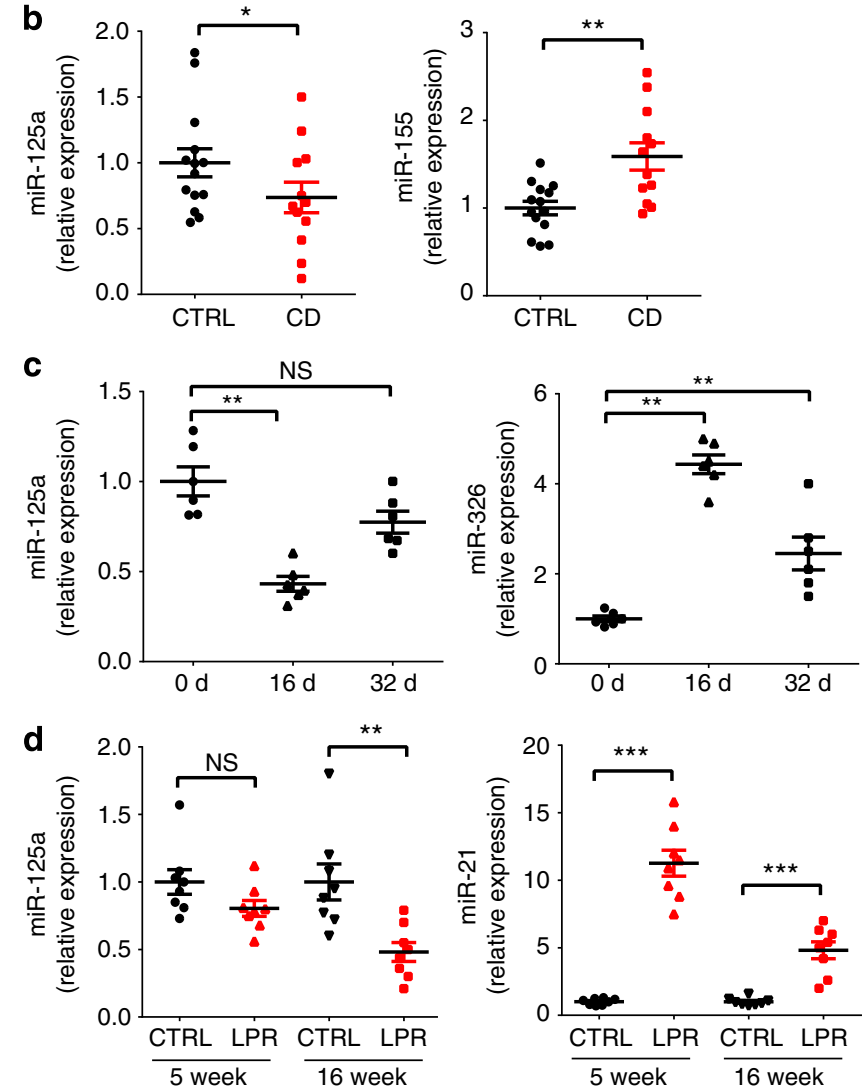

Figure 1 | MiR-125a is downregulated in autoimmune CD4 ${ }^{+} \mathbf{T}$ cells.

(a) Quantitative realtime (RT)-PCR validation of miR-125a expression, with miR-21 as a positive control, in peripheral CD4 ${ }^{+}$T cells from 22 SLE patients and 20 healthy controls (CTRLs). (b) Quantitative RT-PCR validation of miR-125a expression, with miR-155 as a control, in peripheral $\mathrm{CD}^{+}{ }^{+}$T cells from 12 patients with $\mathrm{CD}$ and 14 healthy controls. (c) Quantitative RT-PCR validation of miR-125a expression, with miR-326 as a positive control, in splenic $\mathrm{CD}_{4}{ }^{+} \mathrm{T}$ cells isolated from six naive control C57BL/6 mice, six EAE mice 16 days after immunization or six EAE mice 32 days after immunization. (d) Quantitative RT-PCR validation of miR-125a expression, with miR-21 as a positive control, in splenic CD4 ${ }^{+}{ }^{+}$cells isolated from MRL//pr mice at 5 or 16 weeks of age and normal controls. $n=8$ Mice per group. The relative miRNA expression was normalized to the expression of RNU48 (a,b) or snoRNA202 (c,d). All data are mean \pm s.e.m. ${ }^{\star} P<0.05,{ }^{\star \star} P<0.01,{ }^{\star \star \star} P<0.001$ (nonparametric Mann-Whitney $U$-test). NS, not significant. Data are representative of two $(\mathbf{a}, \mathbf{b})$ or three $(\mathbf{c}, \mathbf{d})$ independent experiments. For additional clinical information, refer to Supplementary Tables 1 and 2. 
(Fig. 1a), 12 patients with Crohn's disease (CD) versus 14 healthy controls (Fig. 1b), myelin olygodendrocyte glycoprotein (MOG)induced EAE versus controls (Fig. 1c) and MRL/lpr mouse strain versus control mice (Fig. 1d). Together, these data suggest that miR-125a may be a commonly downregulated miRNA in multiple human autoimmune diseases and related mouse models.

Treg differentiation is defective in miR-125a ${ }^{-1-} \mathrm{T}$ cells. To further explore the function of miR-125a in autoimmunity, we generated miR-125a transgenic (TG) mice (Supplementary Fig. 2) and knockout (KO) mice (Supplementary Fig. 3). Percentages of $\mathrm{T}\left(\mathrm{CD}^{+}{ }^{+}\right.$or $\left.\mathrm{CD}^{+}\right), \mathrm{B}\left(\mathrm{B} 220^{+}\right)$or myeloid $\left(\mathrm{Mac}-1^{+}\right)$lineages in peripheral lymph nodes or spleens from 6- to 8-week-old miR-125a KO and control wild-type (WT) mice were initially analysed by flow cytometry. The results showed that there were no statistical differences in these lineages between WT and KO mice (Supplementary Table. 3). We next checked whether miR-125a affected CD4 ${ }^{+}$T-cell activation or proliferation. Flow cytometric analysis showed that the percentages of different activation markers in $\mathrm{CD}^{+} \mathrm{T}$ (Supplementary Fig. 4a) or proliferation of carboxyfluorescein succinimidyl ester (CFSE)labelled $\mathrm{CD}^{+}{ }^{+} \mathrm{T}$ cells (Supplementary Fig. $4 \mathrm{~b}$ ) were not significantly altered between KO and WT mice. As a previously reported miRNA profile data have shown that miR-125a was highly expressed in mouse $\mathrm{CD} 4{ }^{+} \mathrm{CD} 25^{+} \mathrm{GITR}^{+}$ Treg cells ${ }^{15}$, we next examined and found that miR-125a was indeed highly expressed in $\mathrm{CD} 4{ }^{+} \mathrm{CD} 25^{\text {hi }}$ population compared with naive T population (Fig. 2a) and was also markedly induced in in vitro-derived Treg cell subset (Fig. 2b). These data implied that miR-125a might have a function in Treg cells. We further examined the percentages of different $\mathrm{T}$-cell subsets in $\mathrm{CD} 4{ }^{+}$ $\mathrm{T}$ population from the spleens of KO or WT mice, and found that the proportion and absolute number of Foxp $3^{+}$Treg cells showed a significant reduction in miR-125a KO mice compared with that in WT mice (Fig. 2c-e), while the other T-cell subsets such as $\mathrm{T}_{\mathrm{H}} 1, \mathrm{~T}_{\mathrm{H}} 2$ and $\mathrm{T}_{\mathrm{H}} 17$ showed no significant differences (Supplementary Table. 3). To confirm the defective differentiation of Treg in miR-125a-deficient mice, we also sorted naive $\mathrm{CD} 4{ }^{+} \mathrm{T}$ cells $\left(\mathrm{CD} 4{ }^{+} \mathrm{CD} 25^{-} \mathrm{CD} 44^{\mathrm{lo}} \mathrm{CD} 62 \mathrm{~L}^{\mathrm{hi}}\right)$ from 6- to 8-week-old miR-125-deficient mice and their WT littermates, cultured the cells under different T-cell subsetskewing conditions for 4 days and then assessed their differentiation efficiency. Flow cytometric analysis revealed that miR-125a-deficient $\mathrm{T}$ cells were indeed impaired in their ability to differentiate into Foxp $3^{+}$population compared with WT T cells, even at a high concentration of transforming growth factor (TGF)- $\beta$ (Fig. 2f). Furthermore, the Foxp3 mRNA level was also significantly decreased in miR-125a-deficient Treg cells relative to that in their WT controls (Fig. 2g). Consistent with the above in vivo observation, flow cytometric analysis showed miR-125a had no obvious effects on in vitro $\mathrm{T}_{\mathrm{H}} 1, \mathrm{~T}_{\mathrm{H}} 2$ and $\mathrm{T}_{\mathrm{H}} 17$ differentiation (Supplementary Fig. 5). Together, these results show that miR-125a-deficient $\mathrm{T}$ cells may be defective in their ability to differentiate into Treg cells, both in vitro and in vivo.

MiR-125a ${ }^{-1-}$ Treg cells fail to prevent colitis effectively. We next tried to examine whether there was any defect in the immunoregulatory capacity of Treg cells. To achieve that, we examined Treg cells from miR-125a-deficient and WT mice in overcoming the immune pathogenesis caused by adoptive transfer of naive $\mathrm{CD}^{+} \mathrm{T}$ cells into immunodeficient $\mathrm{Rag}^{-/-}$mice. Briefly, naive $\mathrm{T}$ cells $\left(\mathrm{CD} 4{ }^{+} \mathrm{CD} 25^{-} \mathrm{CD} 44^{\mathrm{lo}} \mathrm{CD} 62 \mathrm{~L}^{\mathrm{hi}}\right)$ were sorted from C57BL/6 WT mice and Treg cells $\left(\mathrm{CD} 4{ }^{+} \mathrm{CD} 25^{\mathrm{hi}}\right)$ were sorted from WT or miR-125a-deficient mice. Then, naive T cells were transferred into $\mathrm{Rag} 1^{-/}$mice alone or in combination with WT or miR-125a-deficient Treg cells (Fig. 3a). Disease progression was assessed by weight loss within 40 days. We observed that Rag1 ${ }^{-1-}$ mice transferred with WT naive T cells alone gradually developed severe body weight loss. Co-transfer of sufficient numbers of WT Treg cells prevented the disease much more effectively than co-transfer of miR-125a-deficient Treg cells, indicating a defect of Treg cell-mediated suppression in miR-125a-deficient mice (Fig. 3b). Haematoxylin-eosin staining of the colon sections also showed denser infiltration of leukocytes and more severe epithelial hyperplasia in $\mathrm{Rag}^{-/}$mice co-transferred with miR-125a-deficient Treg cells than those co-transferred with WT Treg cells (Fig. 3c). Taken together, these data suggest that miR-125a-deficient Treg cells fail to effectively prevent the immunopathology of T-cell transfer-induced colitis. MiR-125a may contribute to sustain Treg cell-mediated immunosuppressive function.

MiR-125a ${ }^{-/-}$mice are susceptible to autoimmune EAE. To test whether failure to maintain immune homeostasis in the absence of miR-125a was associated with some autoimmune disorders, we induced EAE model in miR-125a-deficient and WT mice. In EAE, Treg cells are required for the suppression of both $\mathrm{T}_{\mathrm{H}} 1$ - and $\mathrm{T}_{\mathrm{H}} 17$-cell subsets ${ }^{16}$. We first evaluated a set of parameters in MOG-induced EAE pathology, and observed that the clinical score and amounts of inflammatory infiltration and demyelination were significantly increased in the miR-125adeficient mice compared with those in their WT controls (Fig. 4a,b). Consistently, the mRNA levels of genes related to $\mathrm{T}_{\mathrm{H}} 1$, such as Ifng, and those related to $\mathrm{T}_{\mathrm{H}} 17$ lineage, such as Il17a, Il17f, Il21 and Il23r, were significantly upregulated in splenic $\mathrm{CD}^{+}{ }^{+} \mathrm{T}$ cell from miR-125a-deficient mice compared with those from WT controls (Fig. 4c). The concentration of interferon (IFN)- $\gamma$ and interleukin-17a (IL-17a) in the supernatants from splenocyte culture challenged with MOG peptide was also increased in miR-125a-deficient mice compared with WT controls (Fig. 4d). Furthermore, intracellular cytokine staining showed the percentage of Treg cells in the CD4 ${ }^{+}$T-cell population isolated from the spleens (Fig. 4e,f) and the central nervous system (CNS) infiltrates (Fig. 4g,h) were significantly decreased in miR-125a-deficient mice compared with those in the WT controls. In contrast, the percentages of inflammatory IFN- $\gamma$ and IL-17a- producing subsets in the $\mathrm{CD} 4{ }^{+}$population were higher in miR-125-deficient mice than that in WT mice in both the spleens (Fig. 4e,f) and the CNS infiltrates (Fig. 4g,h). Consistently, the clinical score of EAE was lower in the miR-125a TG mice than in WT mice (Supplementary Fig. 6a). There were higher levels of in vitro-derived Foxp3 ${ }^{+}$Treg cells and Foxp3 mRNA in miR-125a TG mice than in WT mice (Supplementary Fig. 6b,c). The percentage of $\mathrm{IFN}-\gamma^{+}$was significantly decreased in the $\mathrm{CD} 4{ }^{+} \mathrm{T}$ cells isolated from the CNS of TG mice compared with WT mice (Supplementary Fig. 6d). Collectively, these experiments show that miR-125a-deficient mice are susceptible to autoimmune EAE, manifesting fewer Treg population and greater production of proinflammatory cytokines in the periphery and CNS.

MiR-125a ${ }^{-/-}$Treg cells display increased effector $\mathbf{T}$ genes. To gain insights into the general functional features of miR-125adeficient Treg cells, we sorted $\mathrm{CD} 4{ }^{+} \mathrm{CD} 25^{\text {hi }}$ Treg population from the spleens of 6- to 8-week-old miR-125a-deficient and their littermate WT mice, and then performed a genome-wide genearray analysis. We then predicted the biological functions and mapped the biomolecular networks of the differentially expressed gene transcripts using Ingenuity Pathway Analysis tool. The most significant enrichment of the differentially expressed genes was 

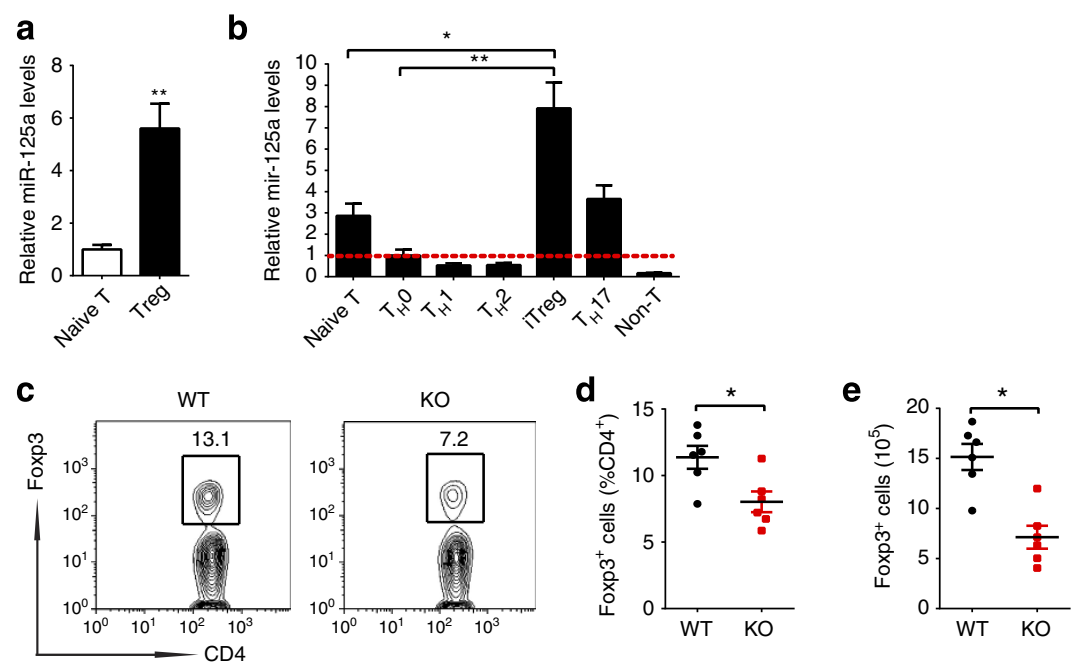

f
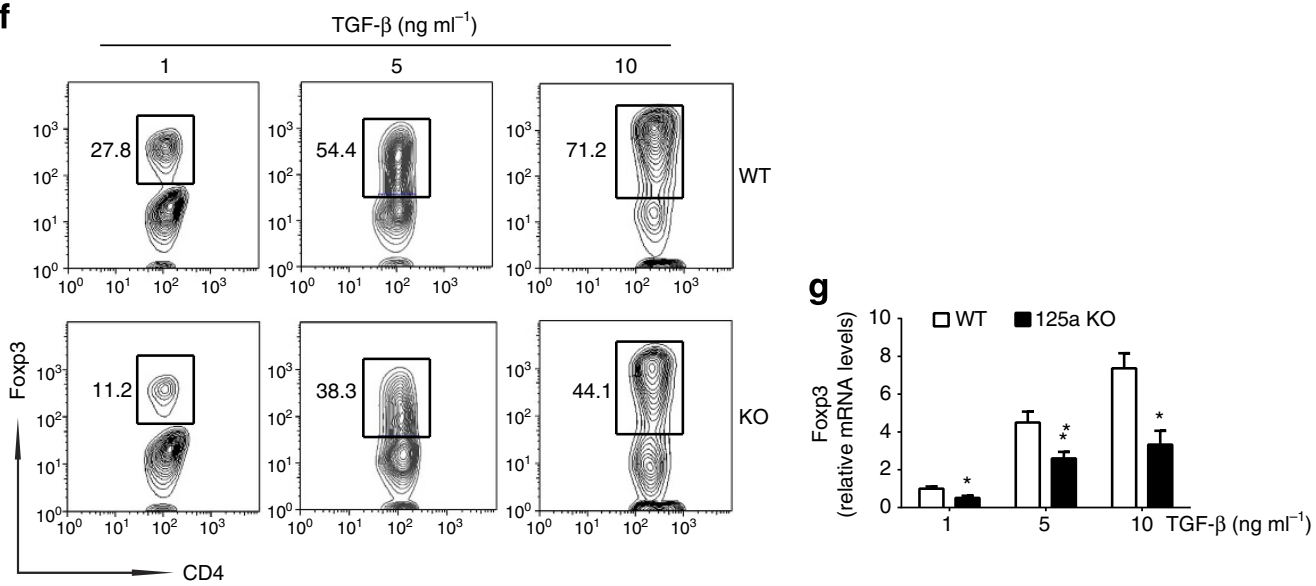

Figure 2 | Treg differentiation is defective in miR-125a- / $-\mathbf{T}$ cells. (a) Naive T cells $\left(C D 4+C D 25^{-}\right.$CD $44^{\text {lo }}$ CD $\left.62^{\text {hi }}\right)$ and Treg cells $\left(C D 4{ }^{+}\right.$CD25 hi $)$ were isolated from the spleens of C57BL/6 mice. Quantitative RT-PCR analysis of miR-125a expression was performed on these two populations.

(b) Quantitative RT-PCR analysis of miR-125a expression in different CD4 ${ }^{+}$T-helper cell subsets differentiated in vitro for 4 days under different skewing conditions. (c) Flow cytometric analysis of Foxp3 ${ }^{+}$Treg cells from the spleens of 6- to 8-week-old miR-125a-deficient mice and their WT littermates. (d) Frequency of Foxp $3^{+}$Treg cells in the $\mathrm{CD} 4^{+}$population in $\mathbf{c}\left(n=6\right.$ per group). (e) Absolute numbers of Foxp3 ${ }^{+}$Treg cells in $\mathbf{c}(n=6$ per group). (f) Flow cytometric analysis of naive $\mathrm{CD} 4{ }^{+} \mathrm{T}$ cells $\left(\mathrm{CD} 4{ }^{+} \mathrm{CD} 25^{-} \mathrm{CD} 44^{\mathrm{lo}} \mathrm{CD} 62 \mathrm{~L}^{\mathrm{hi}}\right)$ isolated from the spleens of 6- to 8-week-old miR-125a-deficient mice and their WT littermates, cultured for 4 days with IL-2 $\left(5 \mathrm{U} \mathrm{ml}^{-1}\right)$, anti-CD3 antibody $\left(2 \mu \mathrm{g} \mathrm{ml}^{-1}\right)$ and anti-CD28 antibody $\left(2 \mu \mathrm{g} \mathrm{ml}{ }^{-1}\right)$, plus various concentrations of TGF- $\beta$. (g) Quantitative RT-PCR analysis of Foxp3 expression in Treg cells differentiated as in $\mathbf{f}$ from miR-125a-deficient mice or their WT littermate controls. The relative miR-125a expression was normalized to the expression of snoRNA202 (a,b), while the relative expression levels of Foxp3 were normalized to the expression of mouse Rpl13a. Data are displayed as mean \pm s.e.m. ${ }^{\star} P<0.05,{ }^{\star \star} P<0.01$ versus control (two-tailed Student's $t$-test). Data are representative of three (a-f) or four $(\mathbf{g})$ independent experiments.

related to T-helper cell pathways. A salient characteristic of Treg cells is their inability to produce proinflammatory cytokines. However, in the absence of miR-125a, Treg cells showed increased expression of genes related to $\mathrm{T}_{\mathrm{H}} 1$-, $\mathrm{T}_{\mathrm{H}}$ 2- and $\mathrm{T}_{\mathrm{H}} 17$ subsets, including some lineage-specific transcriptional factors, cytokines and cytokine receptors. Consistently, Treg lineagespecific transcription factor Foxp3 was decreased, together with some cytokine genes such as $T g f b 1$ (Fig. $5 \mathrm{a}$ ). We further examined the expression of most of the genes cited above with real-time PCR. The results confirmed the array data (Fig. 5b), showing that in the absence of miR-125a, Treg cells showed increased expression of effector lineage genes and reduced expression of Treg lineage genes such as Foxp3. These data also implied that sustained expression of miR-125a in Treg cells may restrain the differentiation programs of effector lineages to stabilize Treg lineage commitment. Although deficiency of miR-125a in Treg cells upregulated $\mathrm{T}_{\mathrm{H}} 1-, \mathrm{T}_{\mathrm{H}} 2$ - and $\mathrm{T}_{\mathrm{H}} 17$-related genes, miR-125a had no obvious effects on in vitro $\mathrm{T}_{\mathrm{H}} 1, \mathrm{~T}_{\mathrm{H}} 2$ and $\mathrm{T}_{\mathrm{H}} 17$ differentiation, as well as in vivo proportions of these $\mathrm{T}$ effector subsets. Therefore, we postulated that the exaggerated proinflammatory cytokine production in miR-125a-deficient mice at pathogenesis conditions such as EAE might be mainly attributable to the break of immune tolerance caused by reduced differentiation or suppressor function of Treg cells, but might not be a cell-intrinsic property of miR-125a-deficient effector $\mathrm{T}$ cells.

Mir-125a inhibits several effector $\mathrm{T}$ lineage factors. The above results raised several questions such as how exactly miR-125a restrained effector lineage programs in Treg cells, and whether the elevated genes in miR-125-deficient Treg cells were the direct targets of miR-125a or the consequence of a secondary effect caused by the reduced expression of Foxp3. To answer these questions, we screened the direct targets of miR-125a by combining target prediction by TargetScan with microarray gene expression data to identify the overlapping and upregulated gene transcripts. Using a combination of these two approaches, we 
a
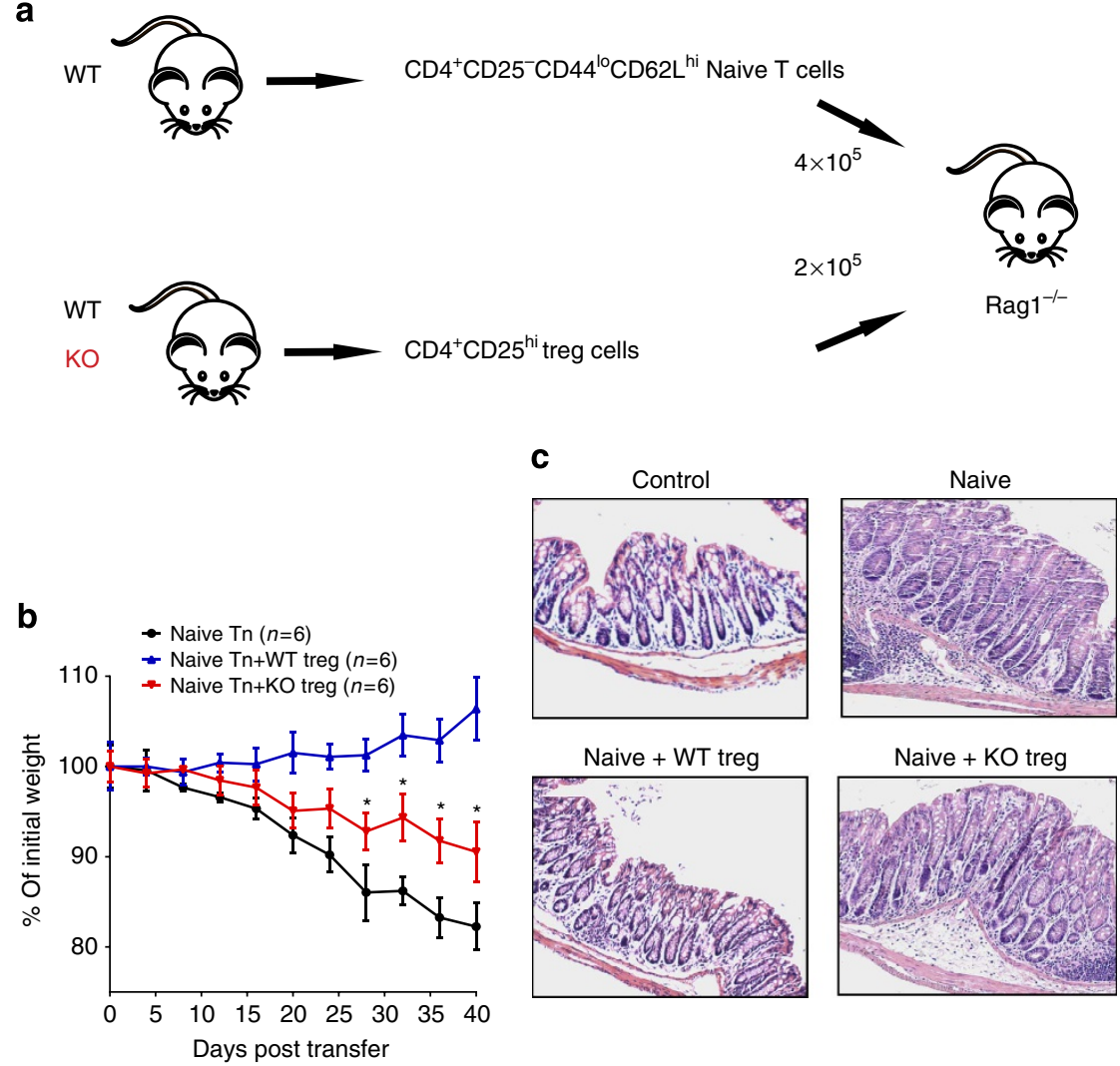

Figure 3 | MiR-125a $\mathbf{a}^{-/}$Treg cells fail to prevent colitis effectively. (a) Schematic illustration of how T-cell transfer model of colitis was investigated. Each Rag $1^{-/-}$recipient mouse was transferred with $5 \times 10^{5} \mathrm{CD} 4{ }^{+} \mathrm{CD} 25^{-} \mathrm{CD} 44^{\mathrm{lo}} \mathrm{CD} 62 \mathrm{~L}^{\text {hi }}$ naive T cells isolated from WT mice, and co-transferred with $2.5 \times 10^{5} \mathrm{CD} 4+{ }^{+} \mathrm{CD} 25^{\text {hi }}$ Treg cells isolated from WT or miR-125a-deficient mice. (b) The weight loss of the mice in a was monitored every 4 days for 40 days from the day after injection. $n=6$ Mice for each group. ${ }^{\star} P<0.05$ (multiple Student's $t$-test). (c) Representative haematoxylin-eosin staining of colon sections from untreated WT mice (control), Rag $1^{-/-}$mice transferred with WT naive T cells (naive), Rag1 $1^{-/-}$mice co-transferred with WT naive T cells and WT Treg cells (naive + WT Treg), and Rag1 ${ }^{-/-}$mice co-transferred with WT naive T cells and miR-125a-deficient Treg cells (naive + KO Treg; original magnification, $\times 20$ ). Data are representative of two independent experiments (mean \pm s.e.m.).

identified 74 genes. Among them, only five predicted genes (Stat3, Tnfsf4, Tnfaip3, Mapkapk2 and Ppp5c) have been reported to have the potential functions in immune responses or pathways (Supplementary Table 4). We confirmed the inhibitory effect using a luciferase reporter assay and found that miR-125a significantly suppressed the luciferase activity of the $3^{\prime}$ untranslated region (UTR) of Stat3 and Tnfsf4 (Supplementary Fig. 7). To avoid potentially significant miR-125a targets from being excluded, we next examined the genes that belonged to T-helper cell pathway and whose expression were inversely correlated to the expression of miR-125a in the array data, including those encoding major transcription factors, cytokines, cytokine receptors and signalling molecules, and focused on 21 potential genes (Supplementary Table 5). We next cloned the $3^{\prime}$ UTRs of all these genes into luciferase reporter constructs, and found that miR125a markedly repressed the reporter activities of several genes, including Stat3, Ill3 and Ifng, whereas the activity of others was either slightly affected or unaffected by miR-125a (Fig. 6a). We further identified the potential binding sites of miR-125a in the $3^{\prime}$ UTRs of Stat3, Il13 and Ifng by TargetScan (Fig. 6b). Mutation of these binding sites confirmed the suppressive roles of miR$125 \mathrm{a}$ on these genes (Fig. 6c). To further demonstrate that Stat3, Il13 and Ifng were the direct targets of miR-125a, we next detected the relative mRNA and protein levels of the targets after overexpression of miR-125a. Since Stat3 is constitutively expressed in some human and mouse cell lines, and the target sites are highly conserved between different species, we first transfected HEK293T cells with two different doses of miR-125a agomirs that are chemically synthesized double-stranded RNA (dsRNA) oligonucleotides used to mimic miRNA. We also infected mouse $\mathrm{CD} 4^{+} \mathrm{T}$ cells with retrovirus-mediated overexpression of miR-125a. The results that showed miR-125a suppressed the protein levels of endogenous Stat3 expression in a dose-dependent manner (Fig. 6d). Considering Il13 and Ifng are secreted cytokines, we next generated retrovirus-based constructs to overexpress miR-125a, and infected $\mathrm{CD}^{+}{ }^{+} \mathrm{T}$ cells under Th1- or Th2-skewing conditions for 4 days, respectively. The infection efficiency, calculated as the percentage of $\mathrm{GFP}^{+}$cells, was $\sim 40 \%$ when the cells were infected once, and $>85 \%$ when they were infected repeatedly (Supplementary Fig. 8). The amounts of IFN- $\gamma$ and IL-13 in the supernatants of the cells infected with miR-125a were significantly reduced compared with that in cells infected with the empty vector (Fig. 6e). The mRNA levels of Stat3, Ifng and Il13 were also significantly reduced after overexpression of miR-125a (Fig. 6f). Considering the fact that Stat3 is a critical transcriptional factor required for $\mathrm{T}_{\mathrm{H}} 17$ differentiation, which inhibits Smad3 binding to enhancer I to limit Treg differentiation ${ }^{17,18}$, Ifng and Il13 are $\mathrm{T}_{\mathrm{H}} 1 / \mathrm{T}_{\mathrm{H}} 2$ polarizers that can also potently inhibit Foxp3 expressing Treg cells differentiation from naive $\mathrm{CD} 4^{+}$precursors ${ }^{19,20}$, these data therefore show that miR-125a directly represses several targets of effector $\mathrm{T}$ lineages that are detrimental to Treg differentiation, which may help to stabilize Treg lineage commitment and function. 
a

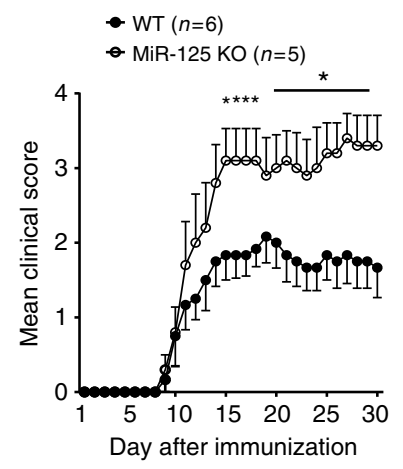

C

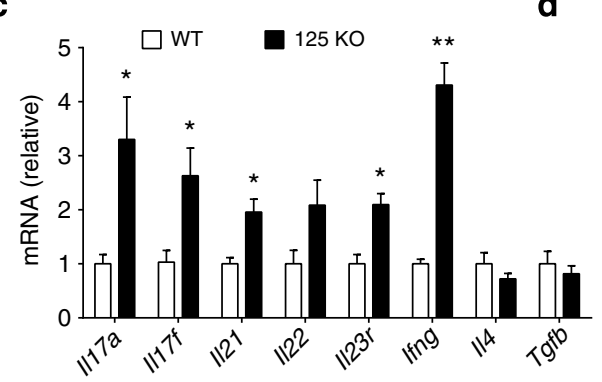

d b
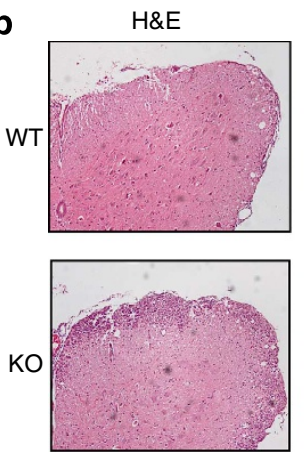

Luxol fast blue
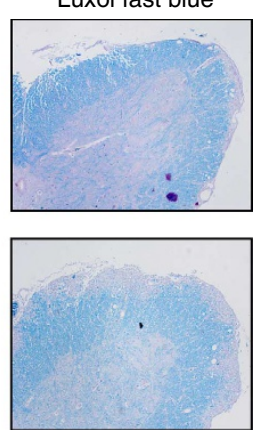

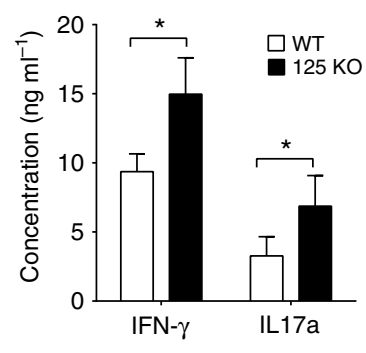

e
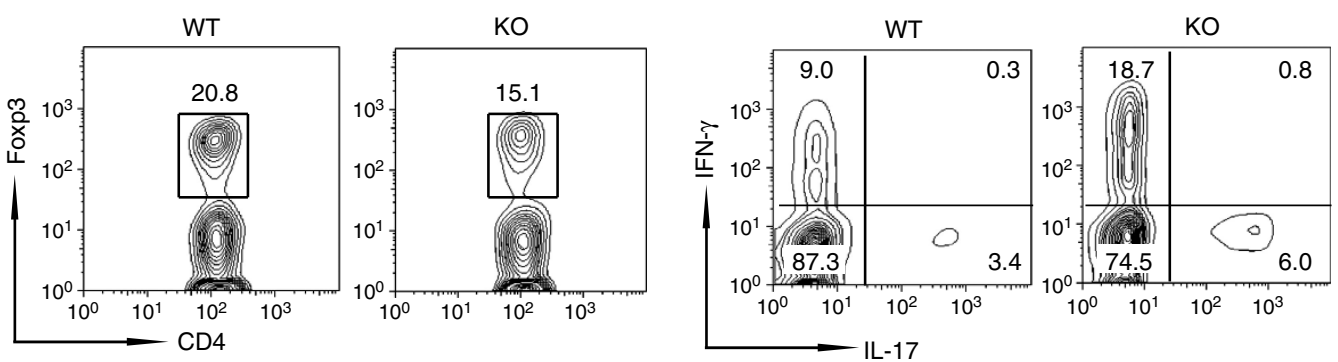

g

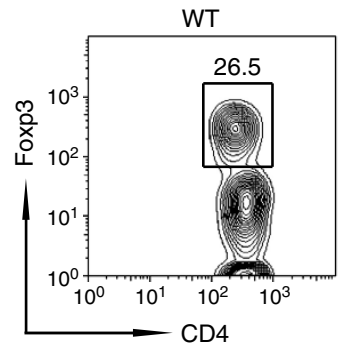

$\mathrm{KO}$
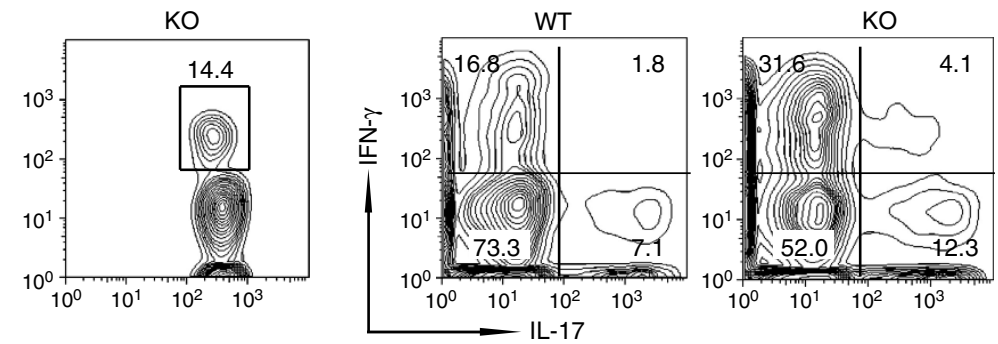

f
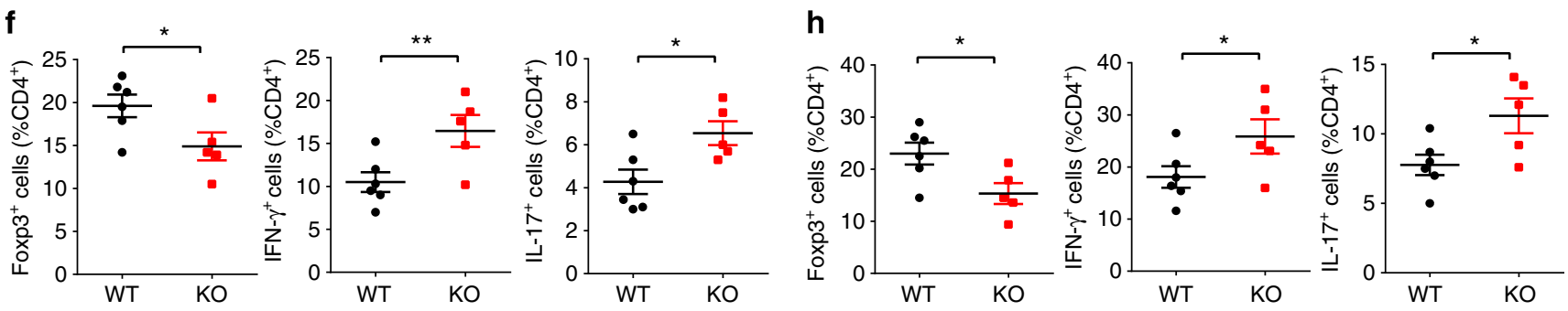

Figure 4 | MiR-125a ${ }^{-/}$mice are susceptible to autoimmune EAE. (a)Mean clinical scores ( \pm s.e.m.) of EAE induced in 6- to 8-week-old WT and miR-125a-deficient mice. (b) Representative haematoxylin-eosin (H\&E) staining or Luxol Fast Blue staining of spinal cord sections on day 21 after immunization of the mice in a. (c) Quantitative RT-PCR analysis of gene expression in splenic CD4 + T-cell samples on day 21 after immunization of the mice in $\mathbf{a}$. The relative expression levels of different genes were normalized to the expression of mouse Rpl13a. (d) Enzyme-linked immunosorbent assay examination of the concentration of IFN- $\gamma$ and IL-17a in the supernatants from the splenocyte culture challenged with MOG peptide. (e,g) Representative flow cytometric analysis of the percentage of Treg, $T_{H} 1$ and $T_{H} 17$ cells in the CD4 ${ }^{+}$T cells isolated from the spleens (e) and CNS (g) of the mice (a) on day 21 after immunization. (f,h) The percentages of Foxp $3^{+}$Treg cells, IFN- $\gamma^{+}$T cells and IL-17 ${ }^{+}$T cells in the CD4 ${ }^{+}$populations from the spleens $(\mathbf{e})$ and CNS (g), respectively. ${ }^{\star} P<0.05,{ }^{\star \star} P<0.01$ versus control (two-tailed Student's $t$-test). Data are representative of three (a,b, e-h) or four (c, d) independent experiments (mean \pm s.e.m.). 
a

\begin{tabular}{|c|c|c|c|c|c|}
\hline \multicolumn{2}{|c|}{ Transcript } & \multicolumn{2}{|c|}{ Secreted moleculars } & \multicolumn{2}{|c|}{ Cell surface molect } \\
\hline Nfat5 & 4.41 & 119 & 11.07 & Il10ra & 4.55 \\
\hline Batf3 & 2.88 & 115 & 9.71 & II17re & 2 \\
\hline Gata3 & 2.67 & $1 / 13$ & 5.60 & Tnfsf4 & 1.6 \\
\hline KIf9 & 2.60 & 114 & 5.36 & II17rb & 1. \\
\hline Batf & 2.57 & 1122 & 5.24 & II17ra & \\
\hline Rorc & 1.54 & 1121 & 4.19 & Icos & \\
\hline Stat3 & 1.41 & 1124 & 2.46 & $C d 28$ & \\
\hline$B c / 6$ & 1.41 & $1 / 17 a$ & 1.98 & Cxcr3 & \\
\hline Stat1 & 0.59 & $1 / 11$ & 1.62 & Tgfbr2 & \\
\hline KIf4 & 0.45 & 116 & 1.57 & & \\
\hline Foxp3 & 0.43 & Ifng & 1.35 & & \\
\hline \multirow[t]{2}{*}{ KIf2 } & 0.38 & $1123 a$ & 1.34 & & \\
\hline & & Tgfb1 & 0.74 & & \\
\hline
\end{tabular}

b

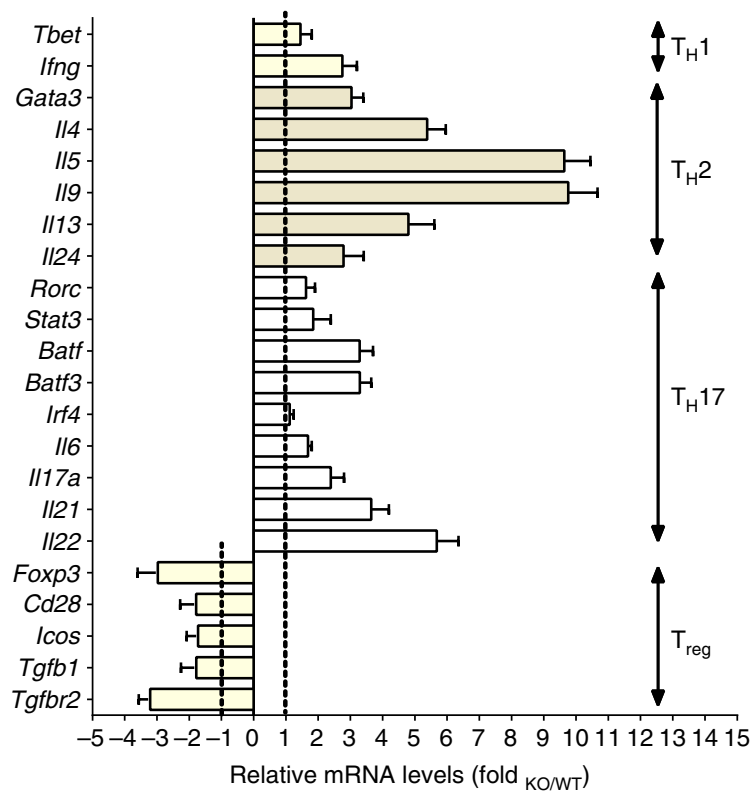

Figure 5 | MiR-125a- / - Treg cells display increased effector T genes. (a) Genome microarray analysis of CD4 ${ }^{+}$CD25 hi Treg cells from miR-125adeficient and WT mice. Splenic CD4 ${ }^{+}$T cells from miR-125a-deficient and WT mice were enriched by Miltenyi Biotec beads. Then, Treg cells $\left(\mathrm{CD} 4{ }^{+}{ }^{\mathrm{CD}} 25^{\text {hi }}\right)$ were sorted by flow cytometry and harvested in Trizol. Samples from three independent experiments were pooled for the microarray analysis. Ingenuity Pathway Analysis analysis was used to investigate which molecular pathways were affected by miR-125a deficiency. Genes that are associated with the T-helper cell pathways show the most significant enrichment, among which gene transcripts up- or downregulated by $>30 \%$ are listed in $\mathbf{a}$. Left, gene symbols. Right, fold change of gene expression in miR-125a-deficient Treg compared with WT Treg. (b) Quantitative RT-PCR analysis of the expression levels of genes associated with T-helper cell differentiation pathway in $\mathbf{a}$. Different T-helper cell subsets are marked in different colours. Data are representative of three (b) independent experiments (mean \pm s.e.m.). Array data are available in GEO under accession code GSE66608.

MiR-125a agomir treatment ameliorates EAE pathogenesis. A series of studies have supported the systemic administration of miRNA as a therapeutic strategy ${ }^{21,22}$. Our current studies showed the suppressive roles of miR-125a on the pathogenesis of autoimmune animal models. Therefore, we sought to evaluate the efficacy and potential application of a miR-125a-based therapy for autoimmune pathogenesis, such as EAE. Agomirs or antagomirs are synthetic RNA molecules with improved stability and delivery, and have been shown to achieve therapeutic overexpression or silencing of an endogenous miRNA gene in vivo ${ }^{23,24}$. To confirm the efficacy of a systemic administration of miR-125a agomir or antagomir in vivo, we first injected mice intravenously with miR-125a agomir, antagomir or their corresponding controls. Different tissues were collected $24 \mathrm{~h}$ after the injection, and miR-125a expression was determined by quantitative PCR. The results showed that miR-125a can be efficiently overexpressed or silenced in most tissues, including the liver, kidney and spleen, but not in the brain, suggesting that agomirs and antagomirs minimally cross the blood-brain barrier (Supplementary Fig. 9). We also used florescence-labelled miR$125 \mathrm{a}$ agomirs to show the systemic transfection efficiency of miRNA into $\mathrm{CD} 4{ }^{+} \mathrm{T}$ cells. Cy5-labelled miR-125a agomirs was first transfected into $\mathrm{CD}^{+} \mathrm{T}$ cells in vitro, and confocal microscopy image showed that the Cy5 fluorescence label was successful (Supplementary Fig. 10a). Cy5-labelled agomirs were further intravenously injected into WT mice for continuous 3 days, and the lymphocytes were harvested 1 day later to test the transfection efficiency. Flow data showed that the transfection efficiency of Cy5-labelled agomir into $\mathrm{CD} 4{ }^{+} \mathrm{T}$ cells in vivo was $\sim 58 \%$ in lymph nodes and $53 \%$ in spleen after three injections (Supplementary Fig. 10b). Since miR-125a sustains Treg cell differentiation and suppressive function during the pathogenesis of EAE, we postulated that treatment with miR-125a agomir could ameliorate the pathogenesis of EAE. As expected, after intravenously injecting a high dose of miR-125a agomir three times at the onset of EAE (within 1 day of initial symptoms), the disease severity was markedly reduced, accompanied by decreased CNS inflammation and demyelination (Fig. 7a,b). Consistently, the percentage of Treg cells was significantly increased $(11.6 \pm 1.08 \%$ versus $18.7 \pm 1.59 \% ; P<0.01$; Fig. $7 \mathrm{c})$, whereas the percentage of IFN- $\gamma^{+}(28.8 \pm 2.82 \%$ versus $20.7 \pm 2.07 \%$, respectively; $P<0.05)$ and $\mathrm{IL}-17^{+}$proinflammatory subsets $(12.8 \pm 1.06 \%$ versus $8.9 \pm 1.05 \%$, respectively; $P<0.05)$ were significantly decreased in the $\mathrm{CD}^{+}{ }^{+} \mathrm{T}$ cells isolated from the CNS after treatment with miR-125a agomirs (Fig. $7 \mathrm{~d}-\mathrm{f}$ ). In contrast, the disease severity of EAE increased significantly with the antagomir-mediated silencing of miR-125a at the onset of EAE (Supplementary Fig. 11), which is consistent with the results of miR-125a-deficient mice. Moreover, to test the role of miR-125 in a therapeutic approach in $\mathrm{EAE}^{25,26}$, we also administered miR-125a agomir for continuous 3 days after acute EAE was established, at a clinical score 1-2. The results showed that miR-125a agomir treatment also significantly ameliorated established EAE symptoms (Fig. $7 \mathrm{~g}$ and Supplementary Fig. 12). Taken together, these data suggest that manipulation of miR-125a expression in vivo not only contributed to EAE prevention but also alleviated established EAE.

\section{Discussion}

The extensive involvement of miRNAs across human diseases helps to understand the pathogenesis of various ailments and lead to therapeutics. However, the promising miRNA candidates for controlling autoimmune pathogenesis are still limited. Our previous work has that shown miR-125a is downregulated in the peripheral $\mathrm{CD}^{+} \mathrm{T}$ cells of SLE patients ${ }^{27}$. Interestingly, miR-125a was also downregulated in oral lichen planus, which is a T-cell-mediated autoimmune disease of oral mucosa ${ }^{28}$. In this study, we further identified miR-125a as a commonly downregulated miRNA in peripheral $\mathrm{CD} 4{ }^{+} \mathrm{T}$ cells of multiple human autoimmune diseases such as SLE and CDs, as well as 


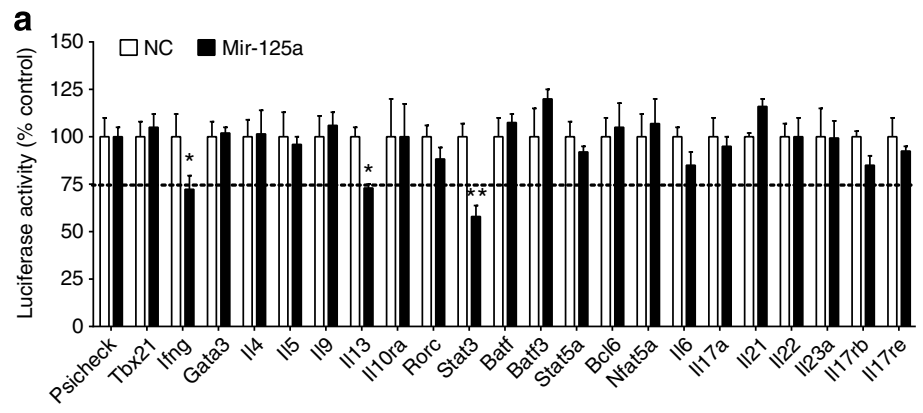

b

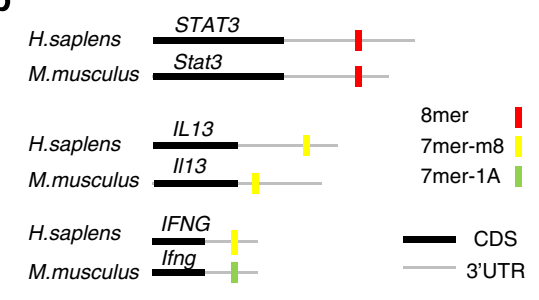

d

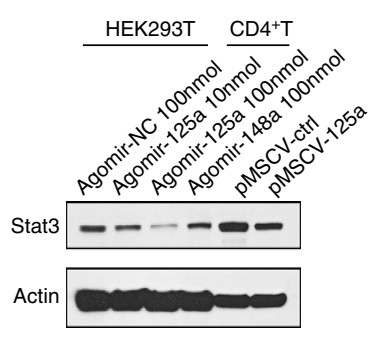

e

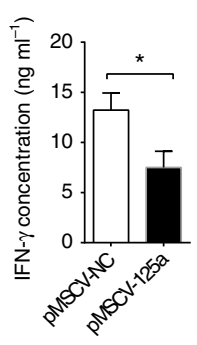

c

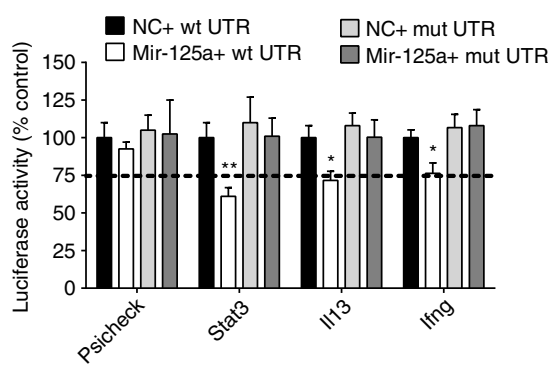

f

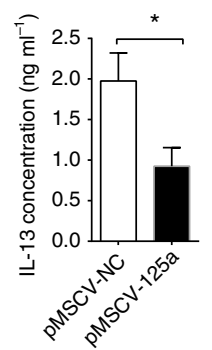

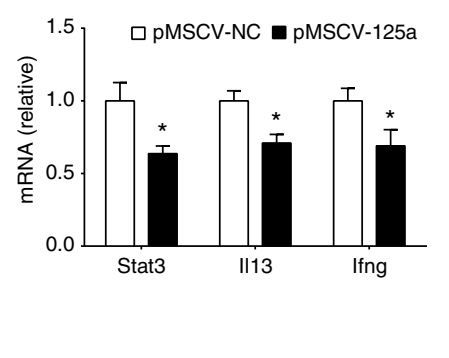

Figure 6 | Mir-125a inhibits several effector T lineage factors. (a) MiR-125a targets Stat3, II13 and Ifng. Dual luciferase assay of HEK293T cells co-transfected with reporter vectors containing the $3^{\prime} U T R s$ of different genes and miR-125a agomir or control agomir. The renilla luciferase activity was normalized to the Firefly luciferase activity. Data are shown as relative luciferase activities of cells transfected with miR-125a related to those of the control cells. (b) Comparison of miR-125a-responsive elements in human and mice transcripts encoding Stat3 (STAT3 and Stat3, respectively), II13 (IL13 and II13, respectively) and Ifng (IFNG and Ifng, respectively) by TargetScan. Colours in the key indicate branch lengths (and match to miR-125a). CDS, coding sequence. (c) Dual luciferase assay of HEK293T cells co-transfected the indicated WT or point-mutated 3'UTR-driven reporter constructs (wt UTR or mut UTR, respectively) with miR-125a or control agomirs. (d) Western blotting of endogenous STAT3 protein levels after transfection of HEK293T cells or infection of $\mathrm{CD}^{+}{ }^{+}$T cells with different doses of miR-125a agomirs or miR-125a retrovirus for 2 days. MiR-148a was used as a control. (e) Enzyme-linked immunosorbent assay examination of IFN- $\gamma$ and IL-13 in the supernatants of CD4 ${ }^{+}$T cells transfected with retrovirus-based miR-125a or empty vector, and differentiated under $\mathrm{T}_{\mathrm{H}}$ 1- or $\mathrm{T}_{\mathrm{H}}$ 2-skewing conditions for 4 days. (f) Quantitative RT-PCR analysis of the mRNA levels of Stat3, II13 and Ifng after the retrovirus-based overexpression of miR-125a in $\mathrm{CD} 4{ }^{+} \mathrm{T}$ cells for 4 days. The relative expression levels were normalized to the expression of mouse $R p / 13 a$. Data are representative of four (a) or three (c-f) independent experiments (mean \pm s.e.m.). ${ }^{\star} P<0.05,{ }^{\star \star} P<0.01$ versus control (two-tailed Student's $t$-test; $\mathbf{a}, \mathbf{e}, \mathbf{f})$ or (one-way analysis of variance and least significant difference $t$-test; $\mathbf{c}$ ).

their related animal disease models. These findings thus provided a very valuable candidate with potential as a biomarker of some autoimmune diseases. Furthermore, we identified miR-125a as a regulator that stabilizes the commitment and immunoregulatory capacity of Treg cells by restraining the differentiation programs of other effector lineages, consequently for the suppression of inflammation. This finding identified miR-125a as a key regulator of $\mathrm{CD}^{+}$T-cell differentiation that prevents autoimmune pathogenesis by controlling the balance between tolerance and autoimmunity.

During the early stage of T-cell differentiation, the cells potentially integrate different environment clues and ultimately allow cells to move towards committing to a particular T-cell lineage ${ }^{29,30}$. The fate decision involves the competition of various transcription factors that dictate different T-cell differentiation programs ${ }^{31-33}$. Recent studies regarding Treg cells development suggests that the decision of antigen-stimulated cells to differentiate into different $\mathrm{T}$ cells subtypes may depend on balancing polarizing cytokines regulated by $\mathrm{T}$ cells subtypespecific transcription factors ${ }^{32,34}$. It has been shown that Foxp3 and Gata3, a specific $\mathrm{T}_{\mathrm{H}} 2$ transcription factor, are able to physically associate with each other to determine T-cell differentiation to either Treg or $\mathrm{T}_{\mathrm{H}} 2$ (ref. 35). Similarly, interaction between Foxp3 and Rorc appears to reciprocally regulate the induction of Treg and $\mathrm{T}_{\mathrm{H}} 17$ cells $^{36-38}$. Foxp3 is able to stabilize the molecular features of the Treg lineage and inhibit effector T-cell developmental factors ${ }^{10,39,40}$. In this study, in the absence of miR-125a, Treg cells showed increased expression of $\mathrm{T}_{\mathrm{H}} 1-, \mathrm{T}_{\mathrm{H}} 2$ - and $\mathrm{T}_{\mathrm{H}} 17$-related effector lineage genes, as well as reduced expression of Treg lineage genes such as Foxp3. As such, miR-125a appears to operate as a crucial regulator in modulating the fate decision of Treg lineage and in the maintenance of immune homeostasis. Further target screen demonstrated that miR-125a directly suppressed several targets, including Stat3, Il13 
a

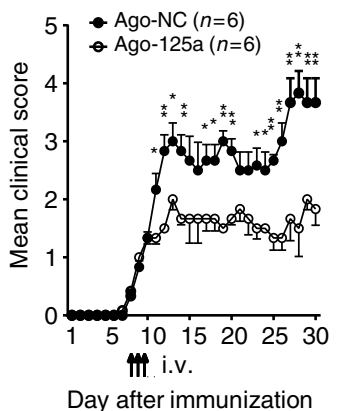

b

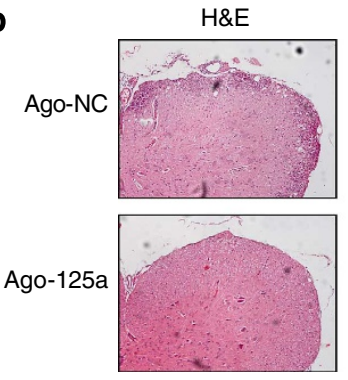

Luxol fast blue

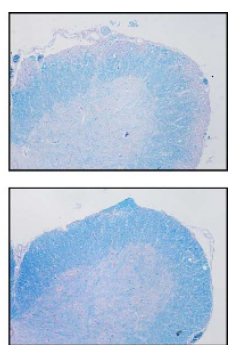

C

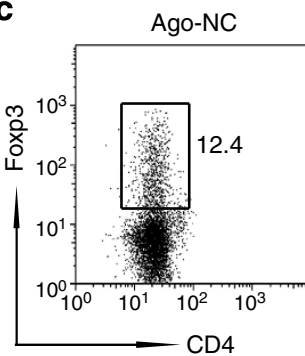

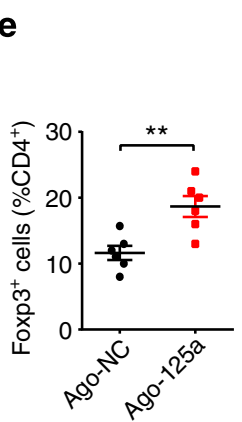

e

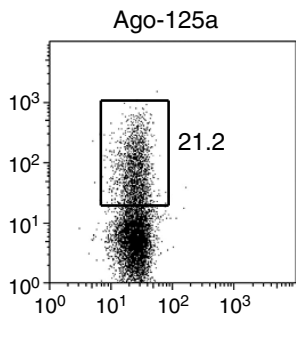

d

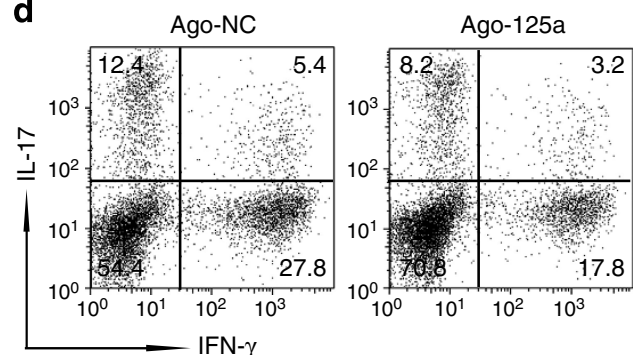

f

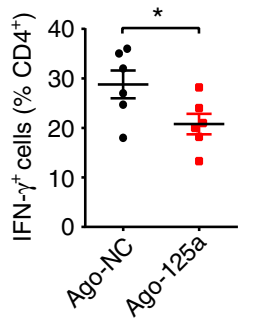

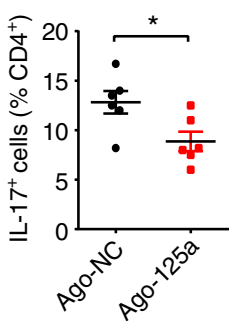

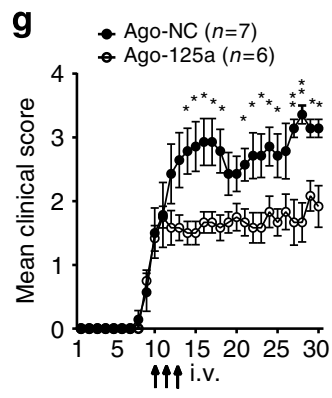

Day after immunization

Figure 7 | MiR-125a agomir treatment ameliorates EAE pathogenesis. (a) Mean clinical scores ( \pm s.e.m.) for EAE induced in 6- to 8-week-old WT mice after three intravenous (i.v.) injections of miR-125a agomir or control agomir ( $5 \mu \mathrm{mol} \mathrm{kg}^{-1}$ per day per mouse) at the onset of EAE. (b) Representative haematoxylin-eosin (H\&E) staining or Luxol Fast Blue staining of spinal cord sections on day 21 after immunization of the mice in a. (c) Flow cytometric analysis of the percentage of Treg cells in the CD4 ${ }^{+}$T cells isolated from the CNS of the mice in a on day 21 after immunization. (d) Flow cytometric analysis of the percentages of $T_{H} 1$ and $T_{H} 17$ in the $C D 4{ }^{+}$T cells isolated from the CNS of the mice (a) on day 21 after immunization. (e) Mean percentages ( \pm s.e.m.) of Foxp3 ${ }^{+}$Treg cells in the $\mathrm{CD}_{4}^{+}$population of $\mathbf{c}\left(n=6\right.$ per group). (f) Mean percentages ( \pm s.e.m.) of IL- $17^{+}$or IFN- $\gamma^{+} \mathrm{T}$ cells in the CD4 ${ }^{+}$ population of $\mathbf{d}$ ( $n=6$ per group). (g) Mean clinical scores ( \pm s.e.m) for EAE induced in 6- to 8-week-old WT mice with three injections of miR-125a agomir ( $5 \mu \mathrm{mol} \mathrm{kg}{ }^{-1}$ per day per mouse) after acute EAE was established, at a clinical score $1-2 . n=7$ or 6 per group. The EAE symptoms were evaluated every day. Data are representative of three (a) or two $(\mathbf{c}-\mathbf{g})$ independent experiments (mean \pm s.e.m.). ${ }^{\star} P<0.05,{ }^{\star \star} P<0.01$ (two-tailed Student's $t$-test).

and Ifng, which are critical factors of effector lineages and also detrimental to Treg differentiation. Thus, miR-125a exerts its roles in a manner partially similar to Foxp3. This finding not only explains how sustained expression of miR-125a in Treg cells is required for restraining effector lineage programs to stabilize Treg lineage commitment and function at a molecular level but also provides insights into the mechanism of miR-125a in controlling immune homeostasis in the context of autoimmunity.

However, although deficiency of miR-125a upregulates some lineage-specific transcriptional factors, cytokines and cytokine receptors in Treg cells, we did not observe obvious effects of miR-125a on $\mathrm{T}_{\mathrm{H}} 1, \mathrm{~T}_{\mathrm{H}} 2$ and $\mathrm{T}_{\mathrm{H}} 17$ differentiation. The potential mechanism may be attributable to the following factors: (1) miR-125a is induced and highly expressed in Treg cells but not in effector T cells, so it is probably that only loss function of miR-125a in Treg cells has obvious effects; (2) miR-125a may inhibit other potential targets that have not been identified yet. miR-125a might have a combinational effect on effector T-cell differentiation, and probably happen to target some negative regulators of $\mathrm{T}_{\mathrm{H}} 1$ and $\mathrm{T}_{\mathrm{H}} 17$ differentiation, in addition to targeting the positive regulators such as Stat 3 and IFN- $\gamma$. Nevertheless, although the absence of miR-125a expression was not associated with a modulation of $\mathrm{T}_{\mathrm{H}} 2$ differentiation in vitro and in vivo on normal conditions, it might be also interesting to test the influence of miR-125a in Th2-prone models such as asthma, in addition to EAE model. Moreover, as we only observed the upregulation of the transcripts of some lineagespecific transcriptional factors, cytokines and cytokine receptors in Treg cells, it also raised a question to measure protein expression of these genes. As Treg cells usually express very low levels of inflammatory cytokines at basal level, a better way is to cross miR-125a KO mice with reporter mice such as Foxp3-Red fluorescent protein (RFP), IL-17-green fluorescent protein (GFP) or IFN- $\gamma$-yellow fluorescent protein (YFP) reporter mice. In that case, the relative expression levels of Foxp3 and effector cytokines such as IL-17 and IFN- $\gamma$ can be easily monitored by the fluorescence intensity of RFP, GFP or YFP under different conditions, such as inflammatory environments. 
Although previous studies have shown that several miRNAs, such as miR-146a (ref. 41), miR-155 (ref. 42) and miR-10a (ref. 43), regulate Treg cells development, suppressor function or homeostasis, the expression patterns of these miRNAs in different autoimmune diseases are still unclear. Our study demonstrated that miR-125a is a commonly dysregulated miRNA in peripheral $\mathrm{CD}^{+} \mathrm{T}$ cells of multiple human autoimmune diseases and animal models. Although the exact mechanisms for the dysregulation of miR-125a in autoimmune pathogenesis are still unknown, we postulate that there might be a potential possibility involved. Previous works have shown that a single-nucleotide polymorphism is located within the precursor $(+8)$ of the mature miR-125a, which significantly blocks the processing of pri-miRNA to pre-miRNA ${ }^{44}$. Whether the genetic polymorphisms within this single locus are associated with numerous autoimmune diseases might be worth further exploring.

Despite the recent discovery of miRNAs, several candidates have already progressed into clinical/preclinical development. The most clinically advanced miRNA-based therapy at the present time is a miR-122 antagonist, SPC3649, that targets an infectious disease-hepatitis C virus-and is currently being evaluated in phase 2 clinical trials ${ }^{45-47}$. Other miRNAs currently in development are in the area of cardiovascular disease and include miR-208 (ref. 48), miR-195 and so on. Our work showed that miR-125a agomir not only contributes to EAE prevention but also alleviates established EAE, thus providing very valuable promise for pharmacological intervention of miR-125a in autoimmune diseases. Although the systemic injection of miR-125a agomir for consecutive three times obtained transfection efficiency $\sim 58 \%$ in lymph nodes and $53 \%$ in spleen, the therapeutic effect of mir-125a agomir on EAE model was quite efficient. One possible reason is that once miR-125a agomir is overexpressed in $\mathrm{T}$ cells, it promotes the differentiation of Treg cells and stabilizes Treg commitment and function, therefore altering the ratio of Treg to $\mathrm{T}$ effector cells in vivo. Treg cells further produce large amount of cytokines such as TGF- $\beta$, which subsequently upregulates miR-125a expression in more $\mathrm{CD}^{+} \mathrm{T}$ cells (Supplementary Fig. 13). Under this condition, mir-125a functions as an initiator and promotes the balance to shift from inflammation to immune suppression through a positive feedback loop.

In summary, our study demonstrates miR-125a as a critical factor that controls autoimmune diseases through stabilizing Treg-mediated immune homeostasis by targeting effector programs. And this finding also provides insights into developing potential miRNA-based therapies in autoimmunity.

\section{Methods}

Mice. MiR-125a KO mice were generated by replacing a 630 -bp-long genome DNA fragment that only encodes mmu-miR-125a with a neomycin selection cassette. The mice were created on the C57BL/6 background by Shanghai Biomodel Organism Science and Technology Development Co., Ltd. The primer sequences used for genotyping were as follows: sense primer $5^{\prime}$-CTGAGGTCTGGATCTCT GGC- $3^{\prime}$ and antisense primer $5^{\prime}$-GCTCAGAAACCCAGTGTCACA- $3^{\prime}$ for WT mice; sense primer $5^{\prime}$-CTGAGGTCTGGATCTCTGGC- $3^{\prime}$ and antisense primer $5^{\prime}$-GCTCAGAAACCCAGTGTCACA- $3^{\prime}$ for KO mice. The mice were crossed with Flp mice to get rid of neomycin gene. Six- to 12 -week-old miR-125a KO mice and their littermate controls were used for experiments. To generate miR-125a TG (miR-125 TG) mice, a 352-bp-long genome DNA fragment encoding mmu-miR$125 \mathrm{a}$ was cloned into the plasmid pSuper, using the forward primer: $5^{\prime}$ -

TCCCTCTTATTCTGGCTTTCTA- $3^{\prime}$ and reverse primer: $5^{\prime}$-GAGGTCAGGT TTCAGTTGGTG- $3^{\prime}$. The mice were created on the C57BL/6 background by Shanghai Biomodel Organism Science and Technology Development Co., Ltd. The primer sequences used for genotyping were as follows: sense primer $5^{\prime}$-GTAAAACGACGGCCAGT- $3^{\prime}$ and antisense primer $5^{\prime}$-AACAGCTATGAC CATG- $3^{\prime}$ on the mouse miR-125a-coding sequence. Six- to 12 -week-old miR-125a $\mathrm{KO}$ or TG mice and their littermate controls were used for experiments. C57BL/6 WT mice were purchased from Shanghai Laboratory Animal Center, Chinese Academy of Sciences. Rag1 ${ }^{-1}$ mice were purchased from Model Animal Research Center of Nanjing University. All mice were maintained in specific pathogen-free conditions. All animal experiments were performed in compliance with the guide for the care and use of laboratory animals, and were approved by the institutional biomedical research ethics committee of the Shanghai Institutes for Biological Sciences (Chinese Academy of Sciences).

Subjects. A total of 34 patients (22 SLE patients and 12 CD patients) were included in the study. Thirty-four normal subjects were matched with the patients for age, sex and race. All participants are from Chinese Han population. The participants with concurrent infections were excluded from the study. For additional clinical information, refer to Supplementary Tables 1 and 2. The study was approved by the Research Ethics Board of Renji Hospital. Peripheral blood samples were collected from each subject in tubes containing acid citrate dextrose formula A.

miRNA and DNA microarray. Total RNA was isolated using TRIzol reagent (Invitrogen). RNA quality was assessed with an Agilent 2100 Bioanalyzer (Agilent), and only samples with an RNA integrity number $>8$ were used. For the miRNA expression analysis, a TaqMan Low Density Array v3.0 (Applied Biosystems) was used to detect and quantified up to 754 human miRNAs or 641 mouse miRNAs using an Applied Biosystems real-time instrument, according to the manufacturer's protocol. Global mRNA expression in iTreg samples from miR-125a-deficient and WT mice were assayed with the Affymetrix GeneChip Mouse Genome 4302.0 Array. Data were further analysed with Agilent GeneSpring GX 10 Microarray Data Analysis Software. Array data are available in GEO under accession code GSE66608.

Reagents. The following antibodies were purchased from BD Pharmingen: antimouse CD4-FITC (GK1.5; used at 1:100 dilution), anti-mouse CD25-APC (PC61; used at 1:200 dilution), anti-mouse CD62L-PE (MEL-14; used at 1:200 dilution), anti-mouse CD44-PE/Cy5 (IM7; used at 1:200 dilution), anti-mouse IFN- $\gamma$-APC (XMG1.2; used at 1:100 dilution) and anti-mouse IL-17-PE (TC11-18H10; used at 1:100 dilution). The following antibodies were purchased from eBioscience: anti-mouse CD3e Functional Grade Purified (145-2C; final concentration used at $2 \mu \mathrm{g} \mathrm{ml}^{-1}$ ), anti-mouse CD28 Functional Grade Purified (37.51; final concentration used at $2 \mu \mathrm{g} \mathrm{ml}^{-1}$ ), anti-mouse/rat Foxp3-PE (FJK-16S; used at 1:100 dilution) and antibody against Stat3 (sc-482; Santa Cruz; used at 1:1,000 dilution).

T-cell purification and activation. Naive $\mathrm{CD} 4{ }^{+} \mathrm{CD} 25^{-} \mathrm{CD} 44^{\mathrm{lo}} \mathrm{CD} 62 \mathrm{~L}^{\text {hi }} \mathrm{T}$ cells were FACS (fluorescence-activated cell sorting) sorted from the spleens of 6- to 8-week-old C57BL/6 mice. FACS-sorted cells were activated with plate-coated anti-CD3 antibody $\left(2 \mu \mathrm{g} \mathrm{ml}^{-1}\right)$ and soluble anti-CD28 antibody $\left(2 \mu \mathrm{g} \mathrm{ml}^{-1}\right)$, and then induced to differentiate into $\mathrm{T}_{\mathrm{H}} 1$ cells by supplementation with IL-12 (10 $\mathrm{ng} \mathrm{ml}^{-1}$; Peprotech) plus anti-IL-4 antibody (11B11; BD Pharmingen), $\mathrm{T}_{\mathrm{H}} 2$ cells with IL-4 (40 $\mathrm{ng} \mathrm{ml}^{-1}$; Peprotech) plus anti-IFN- $\gamma$ antibody (XMG1.2; BD Pharmingen), $\mathrm{T}_{\mathrm{H}} 17$ differentiation, cells usually received anti-IL- 4 and anti-IFN- $\gamma$ plus a $\mathrm{T}_{\mathrm{H}} 17$ 'cocktail', containing TGF- $\beta 1$ ( $2 \mathrm{ng} \mathrm{ml}^{-1}$; R\&D), IL-6 (30 $\mathrm{ng} \mathrm{ml}^{-1}$; Peprotech), tumour necrosis factor (10 $\mathrm{ng} \mathrm{ml}^{-1}$; Peprotech), IL- $1 \beta\left(10 \mathrm{ng} \mathrm{ml}^{-1}\right.$; Peprotech) and IL-23 (20 ng ml ${ }^{-1}$; Peprotech), inducible Treg cells with TGF- $\beta 1$ $\left(5 \mathrm{ng} \mathrm{ml}^{-1} ; \mathrm{R} \& \mathrm{D}\right)$ and recombinant mouse IL-2 (50 $\mathrm{U} \mathrm{ml}^{-1}$; Peprotech). Neutralizing anti-IFN- $\gamma$ and anti-IL- 4 were both used at a concentration of $10 \mu \mathrm{g} \mathrm{ml}^{-1}$. Cells stimulated under 'neutral' conditions (anti-IL-4 plus anti- IFN- $\gamma$, but no additional cytokines) were considered ' $\mathrm{T}_{\mathrm{H}} 0$ ' cells.

Intracellular staining and flow cytometry. For intracellular cytokine staining, cells obtained from in vitro culture or from spleens or CNS infiltrates of mice with EAE were incubated in a tissue culture incubator for $5 \mathrm{~h}$ at $37^{\circ} \mathrm{C}$ with phorbol 12 -myristate 13 -acetate ( $50 \mathrm{ng} \mathrm{ml}^{-1}$; Sigma), ionomycin $\left(750 \mathrm{ng} \mathrm{ml}^{-1}\right.$; Sigma) and brefeldin A $\left(10 \mu \mathrm{g} / \mathrm{ml}^{-1}\right.$; Sigma). Surface staining was performed as described above with the corresponding fluorescence-labelled surface antibodies. After surface staining, the cells were resuspended in fixation/permeabilization solution (Cytofix/Cytoperm Kit; BD Pharmingen), and intracellular cytokine staining was performed according to the manufacturer's protocol. For Foxp3 staining, the cells were not stimulated with phorbol 12-myristate 13-acetate and ionomycin, instead the cells were stained according to the manufacturer's protocol (Foxp3 Staining Buffer Set, eBioscience). FACS Calibur (BD Biosciences) and FlowJo software were used for analysis. Sample data were acquired by FACS Calibur cytometer (BD Biosciences) and analysed using FlowJo software.

Transfer model of colitis. Naive $\mathrm{T}$ cells $\left(\mathrm{CD} 4{ }^{+} \mathrm{CD} 25^{-} \mathrm{CD} 44^{\mathrm{lo}} \mathrm{CD} 62 \mathrm{~L}^{\text {hi }}\right)$ from $\mathrm{C} 57 \mathrm{BL} / 6$ mice were sorted by FACS and then transferred into Rag1 $1^{-/-}$mice alone or in combination with $\mathrm{CD} 4{ }^{+} \mathrm{CD} 25^{\text {hi }}$ Treg cells. After T-cell reconstitution, the mice were weighed weekly and monitored for signs of disease. Mice were killed at 6 weeks after T-cell transfer and their colons were subjected to histopathological analysis. Epithelial cell hyperplasia and leukocyte infiltration were mainly evaluated. 
EAE. The induction of EAE was described previously ${ }^{49,50}$. Briefly, C57BL/6 mice at 6-8 weeks of age were induced by a subcutaneous immunization in the back region with $300 \mu \mathrm{g}$ of the MOG peptide (35-55) in Complete Freund's Adjuvant (CFA; Difco). Pertussis toxin (List Biological Laboratories, Inc.) at a dose of $200 \mu \mathrm{g}$ per mouse in PBS was administered intraveously on the day of immunization and once more $48 \mathrm{~h}$ later. The disease score was evaluated as previously described (ref. 49): 0, no clinical signs; 1, limp tail; 2, paraparesis (weakness, incomplete paralysis of one or two hind limbs); 3, paraplegia (complete paralysis of two hind limbs);

4 , paraplegia with fore limb weakness or paralysis and 5 , moribund state or death. For histology, spinal cords were removed from MOG-immunized mice and were immediately fixed in $4 \%$ paraformaldehyde. Paraffin-embedded $5-\mu \mathrm{m}$ sections of spinal cord were stained with haematoxylin-eosin or Luxol Fast Blue and then examined by light microscopy.

Quantitative PCR. Total RNA was extracted from the cells and mouse tissues with TRIzol reagent (Invitrogen) according to the manufacturer's instructions. cDNA was synthesized with PrimeScript RT reagent kit (Takara). The expression of the genes encoding mouse Foxp3, Tgfb1, Stat3, Rorc, Il17a, Il17f, Gata3, Il4, Il5, Il13, Ifng, Il21, Il22, Il23r and so on were quantified by real-time PCR using the SYBR Premix ExTaq kit (Takara) (sequence data are available upon request). All gene expression results were normalized to the expression of the housekeeping gene Rpl13a. Amplification of cDNA was performed on an AbiPrism 7900 HT cycler (Applied Biosystems). Mature miR-125a and other miRNAs were assayed with a specific TaqMan kit from Applied Biosystems, and their expression was normalized to the expression of snoRNA202 or RNU48.

MiRNA agomir and antagomir. MiR-125a agomir and antagomir were purchased from Ruibo Biotech. MiRNA agomirs are chemically synthesized dsRNA oligonucleotides used to mimic miRNA. The terminal nucleotides at both ends of the dsRNAs are modified by an O-methyl moiety at the $2^{\prime}$-ribose position. Another modification is cholesteryl functionality at the $5^{\prime}$ end of the RNA at sense strand. MiRNA antagomirs are single-stranded oligonucleotides used to silence endogenous miRNAs. Similarly, different chemical modifications are used to enhance the stability and cellular uptake, including partial phosphorothioate backbone modifications, fully $2^{\prime}$-OMe sugar modifications and $3^{\prime}$-cholesterol conjugation. Universal negative controls for both agomirs and antagomirs are based on the sequences of cel-miR-67 in Caenorhabditis elegans, which has been confirmed to have minimal sequence identity with miRNAs in human, mouse or rat.

Luciferase assays. For the $3^{\prime}$ UTR reporter assay, psiCHECK- 2 vector (Promega) was used to clone the $3^{\prime} \mathrm{UTR}$ s of all the predicted targets of miR-125a. Site-specific mutants were generated by PCR in psiCHECK-2 vector. HEK293T cells were seeded in the wells of a 96-well plate 1 day before transfection, and then each well was transfected with a mixture of $10 \mathrm{ng} 3^{\prime} \mathrm{UTR}$ luciferase reporter vector and $0.5 \mu \mathrm{l}$ of $20 \mu \mathrm{M}$ miRNA agomirs. Twenty-four hours after transfection, the cells were lysed and the luciferase activity was measured. The ratio of renilla luciferase to firefly luciferase was calculated for each well.

Immunoblots. Lysates were resolved by electrophoresis, transferred to polyvinylidene fluoride (PVDF) membrane and probed with antibodies directed against Stat3 or Actin. Images have been cropped for presentation in Fig.6d. Full-size images are presented in Supplementary Fig.14

Statistics. The nonparametric Mann-Whitney $U$-test was applied for the statistical comparison of health and patient samples. A two-tailed Student's $t$-test was applied for statistical comparison of two groups or where appropriate. The one-way analysis of variance and least significant difference $t$-test was used for comparison of multiple groups. $P$ values $<0.05$ were considered statistically significant.

\section{References}

1. Gregersen, P. K. \& Olsson, L. M. Recent advances in the genetics of autoimmune disease. Annu. Rev. Immunol. 27, 363-391 (2009).

2. Baumgarth, N. \& Bevins, C. L. Autoimmune disease: skin deep but complex. Nature 449, 551-553 (2007).

3. Nanda, S. Genetic variants suggest shared pathwaysto autoimmune disease. Nat. Rev. Rheumatol. 6, 675 (2010).

4. Littman, D. R. \& Rudensky, A. Y. Th17 and regulatory T cells in mediating and restraining inflammation. Cell 140, 845-858 (2010).

5. Haas, J. et al. Prevalence of newly generated naive regulatory T cells (Treg) is critical for Treg suppressive function and determines Treg dysfunction in multiple sclerosis. J. Immunol. 179, 1322-1330 (2007).

6. Xavier, R. J. \& Podolsky, D. K. Unravelling the pathogenesis of inflammatory bowel disease. Nature 448, 427-434 (2007).

7. Brusko, T. \& Atkinson, M. Treg in type 1 diabetes. Cell Biochem. Biophys. 48, 165-175 (2007).
8. Zheng, Y. et al. Genome-wide analysis of Foxp 3 target genes in developing and mature regulatory T cells. Nature 445, 936-940 (2007).

9. Josefowicz, S. Z. \& Rudensky, A. Control of regulatory T cell lineage commitment and maintenance. Immunity 30, 616-625 (2009).

10. Samstein, R. M. et al. Foxp3 exploits a pre-existent enhancer landscape for regulatory T cell lineage specification. Cell 151, 153-166 (2012).

11. Mendell, J. T. \& Olson, E. N. MicroRNAs in stress signaling and human disease. Cell 148, 1172-1187.

12. van Rooij, E., Purcell, A. L. \& Levin, A. A. Developing microRNA therapeutics. Circ. Res. 110, 496-507 (2012).

13. Jackson, A. L. \& Levin, A. A. Developing microRNA therapeutics: approaching the unique complexities. Nucleic Acid Ther. 22, 213-225 (2012).

14. Jernas, M. et al. MicroRNA regulate immune pathways in T-cells in multiple sclerosis (MS). BMC Immunol. 14, 32 (2013).

15. Cobb, B. S. et al. A role for Dicer in immune regulation. J. Exp. Med. 203, 2519-2527 (2006).

16. Lock, C. et al. Gene-microarray analysis of multiple sclerosis lesions yields new targets validated in autoimmune encephalomyelitis. Nat. Med. 8, 500-508 (2002).

17. Laurence, A. et al. STAT3 transcription factor promotes instability of nTreg cells and limits generation of iTreg cells during acute murine graft-versus-host disease. Immunity 37, 209-222 (2012).

18. Xu, L. L. et al. Positive and negative transcriptional regulation of the Foxp3 gene is mediated by access and binding of the Smad3 protein to enhancer I. Immunity 33, 313-325 (2010).

19. Chang, J. H., Kim, Y. J., Han, S. H. \& Kang, C. Y. IFN-gamma-STAT1 signal regulates the differentiation of inducible Treg: potential role for ROS-mediated apoptosis. Eur. J. Immunol. 39, 1241-1251 (2009).

20. Wei, J. et al. Antagonistic nature of T helper 1/2 developmental programs in opposing peripheral induction of Foxp3 + regulatory T cells. Proc. Natl Acad. Sci. USA 104, 18169-18174 (2007).

21. Kota, J. et al. Therapeutic microRNA delivery suppresses tumorigenesis in a murine liver cancer model. Cell 137, 1005-1017 (2009).

22. Lanford, R. E. et al. Therapeutic silencing of microRNA-122 in primates with chronic hepatitis C virus infection. Science 327, 198-201 (2010).

23. Krutzfeldt, J. et al. Silencing of microRNAs in vivo with 'antagomirs'. Nature 438, 685-689 (2005).

24. Hou, J. et al. Identification of miRNomes in human liver and hepatocellular carcinoma reveals miR-199a/b-3p as therapeutic target for hepatocellular carcinoma. Cancer Cell 19, 232-243 (2011).

25. Weber, M. S. et al. Type II monocytes modulate T cell-mediated central nervous system autoimmune disease. Nat. Med. 13, 935-943 (2007).

26. Youssef, S. et al. The HMG-CoA reductase inhibitor, atorvastatin, promotes a Th2 bias and reverses paralysis in central nervous system autoimmune disease Nature 420, 78-84 (2002).

27. Zhao, X. et al. MicroRNA-125a contributes to elevated inflammatory chemokine RANTES levels via targeting KLF13 in systemic lupus erythematosus. Arthritis Rheum. 62, 3425-3435 (2010).

28. Hu, J. Y. et al. Increasing CCL5/CCR5 on CD4 + T cells in peripheral blood of oral lichen planus. Cytokine 62, 141-145 (2013).

29. Maldonado, R. A., Irvine, D. J., Schreiber, R. \& Glimcher, L. H. A role for the immunological synapse in lineage commitment of CD4 lymphocytes. Nature 431, 527-532 (2004).

30. O'Shea, J. J. \& Paul, W. E. Mechanisms underlying lineage commitment and plasticity of helper CD4 + T cells. Science 327, 1098-1102 (2010).

31. O'Shea, J. J. \& Paul, W. E. Mechanisms underlying lineage commitment and plasticity of helper CD4(+) T cells. Science 327, 1098-1102 (2010).

32. Vahedi, G. et al. STATs shape the active enhancer landscape of $\mathrm{T}$ cell populations. Cell 151, 981-993 (2012).

33. Nakayamada, S., Takahashi, H., Kanno, Y. \& O'Shea, J. J. Helper T cell diversity and plasticity. Curr. Opin. Immunol. 24, 297-302 (2012).

34. Wan, Y. Y. \& Flavell, R. A. Regulatory T-cell functions are subverted and converted owing to attenuated Foxp3 expression. Nature 445, 766-770 (2007)

35. Dardalhon, V. et al. IL-4 inhibits TGF-beta-induced Foxp3 $+\mathrm{T}$ cells and, together with TGF-beta, generates IL-9 + IL $-10+$ Foxp3( - ) effector T cells. Nat. Immunol. 9, 1347-1355 (2008).

36. Bettelli, E. et al. Reciprocal developmental pathways for the generation of pathogenic effector TH17 and regulatory T cells. Nature 441, 235-238 (2006).

37. Zhou, L. et al. TGF-beta-induced Foxp3 inhibits T(H)17 cell differentiation by antagonizing RORgammat function. Nature 453, 236-240 (2008).

38. Ichiyama, K. et al. Foxp3 inhibits RORgammat-mediated IL-17A mRNA transcription through direct interaction with RORgammat. J. Biol. Chem. 283, 17003-17008 (2008).

39. Rudra, D. et al. Transcription factor Foxp3 and its protein partners form a complex regulatory network. Nat. Immunol. 13, 1010-1019 (2012).

40. Rudensky, A. Y. Regulatory T cells and Foxp3. Immunol. Rev. 241, 260-268 (2011). 
41. Lu, L. F. et al. Function of miR-146a in controlling Treg cell-mediated regulation of Th1 responses. Cell 142, 914-929 (2010).

42. Lu, L. F. et al. Foxp3-dependent microRNA155 confers competitive fitness to regulatory T cells by targeting SOCS1 protein. Immunity 30, 80-91 (2009).

43. Takahashi, H. et al. TGF-beta and retinoic acid induce the microRNA miR-10a, which targets Bcl-6 and constrains the plasticity of helper T cells. Nat. Immunol. 13, 587-595 (2012).

44. Duan, R., Pak, C. \& Jin, P. Single nucleotide polymorphism associated with mature miR-125a alters the processing of pri-miRNA. Hum. Mol. Genet. 16, 1124-1131 (2007).

45. Jopling, C. L., Yi, M., Lancaster, A. M., Lemon, S. M. \& Sarnow, P. Modulation of hepatitis C virus RNA abundance by a liver-specific MicroRNA. Science 309, 1577-1581 (2005).

46. Gebert, L. F. R. et al. Miravirsen (SPC3649) can inhibit the biogenesis of miR-122. Nucleic Acids Res. 42, 609-621 (2014).

47. Janssen, H. L. et al. Treatment of HCV infection by targeting microRNA. N. Engl. J. Med. 368, 1685-1694 (2013).

48. van Rooij, E. et al. Control of stress-dependent cardiac growth and gene expression by a microRNA. Science 316, 575-579 (2007).

49. Zhu, S. et al. Modulation of experimental autoimmune encephalomyelitis through TRAF3-mediated suppression of interleukin 17 receptor signaling. J. Exp. Med. 207, 2647-2662.

50. Zhu, S. et al. The microRNA miR-23b suppresses IL-17-associated autoimmune inflammation by targeting TAB2, TAB3 and IKK-alpha. Nat. Med. 18, 1077-1086 (2012).

\section{Acknowledgements}

We thank Robert Georgantas and Philip Brohawn for their helpful discussions, technical expertise and/or review of this manuscript. This work is supported by grants from 973 programme (2014CB541902 and 2014CB541901), National Natural Science Foundation of China (81230072, 81421001, 81025016 and 31370880), the Key Research Program of the Chinese Academy of Sciences (KSZD-EW-Z-003-3), the grants from the State Key Laboratory of Oncogenes and Related Genes (No.91-14-05) as well as Chinese Ministry of Health (201202008) and the Program of the Shanghai Commission of Science and Technology (12431900703, 12JC1406000 and 12ZR1435900). W.P. is supported by a fellowship from Leukemia and Lymphoma Society. S.Z. is supported by a fellowship from Howard Hughes Medical Institute-The Helen Hay Whitney Foundation and Shenzhen Foundation of Science and Technology (grant number GJHZ2014041417082192).

\section{Author contributions}

W.P. and N.S designed the research; N.S. and Y.Y supervised the research; W.P., S.Z. and D.D. conducted the experiments; W.P. wrote the manuscript; N.S, Y.Y and Z.L. revised the manuscript; Y.C. and J.F provided clinical samples; D.L., B.L., Y.Z., Y.T., M.T.W., W.Z., Y.W., B.C. and Y.Q. helped the experiments; J.B.H., R.A.F., N.G., R.H., L.R. and B.J. commented on the research.

\section{Additional information}

Accession codes: Array data are available in GEO under accession code GSE66608.

Supplementary Information accompanies this paper at http://www.nature.com/ naturecommunications

Competing financial interests: The authors declare no competing financial interests.

Reprints and permission information is available online at http://npg.nature.com/ reprintsandpermissions/

How to cite this article: Pan, W. et al. MiR-125a targets effector programs to stabilize Treg-mediated immune homeostasis. Nat. Commun. 6:7096 doi: 10.1038/ncomms8096 (2015). 